\title{
Prognostic Impact of miR-224 and RAS Mutations in Medullary Thyroid Carcinoma
}

\author{
Elisabetta Cavedon, ${ }^{1}$ Susi Barollo, ${ }^{2}$ Loris Bertazza, ${ }^{2}$ Gianmaria Pennelli, ${ }^{3}$ \\ Francesca Galuppini, ${ }^{3}$ Sara Watutantrige-Fernando, ${ }^{2}$ Simona Censi, ${ }^{2}$ Maurizio Iacobone, ${ }^{4}$ \\ Clara Benna, ${ }^{4}$ Federica Vianello, ${ }^{5}$ Stefania Zovato, ${ }^{1}$ Davide Nacamulli, ${ }^{2}$ and Caterina Mian ${ }^{2}$ \\ ${ }^{1}$ Familial Tumor Unit, Veneto Institute of Oncology, (IOV)-IRCCS, Padova, Italy \\ ${ }^{2}$ Endocrinology Unit, Department of Medicine (DIMED), University of Padua, Padua, Italy \\ ${ }^{3}$ Surgical Pathology \& Cytopathology Unit, Department of Medicine (DIMED), University of Padua, Padua, Italy \\ ${ }^{4}$ Surgery Unit, Department of Surgical, Oncological and Gastroenterological Sciences (DISCOG), University of Padua, Padua, Italy \\ ${ }^{5}$ Department of Radiotherapy, Veneto Institute of Oncology (IOV)-IRCCS, Padova, Italy
}

Correspondence should be addressed to Elisabetta Cavedon; elisabettacavedon@yahoo.it

Received 12 January 2017; Revised 17 March 2017; Accepted 11 April 2017; Published 6 June 2017

Academic Editor: Diego Russo

Copyright (c) 2017 Elisabetta Cavedon et al. This is an open access article distributed under the Creative Commons Attribution License, which permits unrestricted use, distribution, and reproduction in any medium, provided the original work is properly cited.

Little is known about the function of microRNA-224 (miR-224) in medullary thyroid cancer (MTC). This study investigated the role of miR-224 expression in MTC and correlated it with mutation status in sporadic MTCs. A consecutive series of 134 MTCs were considered. Patients had a sporadic form in $80 \%$ of cases (107/134). In this group, REarranged during transfection $(R E T)$ and rat sarcoma $(R A S)$ mutation status were assessed by direct sequencing in the tumor tissues. Quantitative real-time polymerase chain reaction was used to quantify mature hsa-miR-224 in tumor tissue. RAS (10/107 cases, 9\%) and RET (39/107 cases, 36\%) mutations were mutually exclusive in sporadic cases. miR-224 expression was significantly downregulated in patients with the following: high calcitonin levels at diagnosis $(p=0.03, r=-0.3)$; advanced stage $(p=0.001)$; persistent disease $(p=0.001)$; progressive disease $(p=0.002)$; and disease-related death $(p=0.0001)$. We found a significant positive correlation between miR-224 expression and somatic RAS mutations $(p=0.007)$. Patients whose MTCs had a low miR-224 expression tended to have a shorter overall survival $(\log$-rank test $p=0.005)$. On multivariate analysis, miR-224 represented an independent prognostic marker. Our data indicate that miR-224 is upregulated in RAS-mutated MTCs and in patients with a better prognosis and could represent an independent prognostic marker in MTC patients.

\section{Introduction}

Medullary thyroid carcinoma (MTC) is a rare neuroendocrine cancer originating from parafollicular, calcitonin(Ct-) producing C-cells. It accounts for 5-10\% of all thyroid carcinomas with a global 10 -year survival rate around $65 \%$ to $70 \%$. About $75 \%$ of MTCs are sporadic, while the remainders are hereditary, due to germline mutations that activate the REarranged during transfection (RET) proto-oncogene [1]. Various clinical, pathological, and genetic variables have been proposed as prognostic markers, including serum calcitonin (Ct) levels at diagnosis, extent of nodal disease, distant metastasis, pathological stage, and mutational damage in tumor suppressor genes [2]. Distinctive germinal RET mutations in the inherited forms and somatic RET mutations in sporadic cases represent the most important molecular markers for an adequate prognostic stratification of MTC patients [3-6].

It has been demonstrated that a combined analysis of somatic RET and Ki-67 is useful for identifying patients with 
a more aggressive cancer, and their joint assessment could ameliorate the initial risk stratification of patients with sporadic MTC, and thus be of prognostic relevance [7].

Moura et al. recently reported finding RAS somatic mutations in $68 \%$ of $R E T$-negative sporadic MTCs. RAS mutations were detected only in $H$-RAS and $K-R A S$ genes (not in $N-R A S$ genes), apparently representing an alternative genetic event to RET mutations in sporadic MTC. These results were reproduced by others, who found proportion $R A S$-mutated cases ranging from $0 \%$ to $26.2 \%$ of MTCs. According to a recent analysis conducted by Ciampi et al. on one of the largest series in the literature [8], the average prevalence of $R A S$ mutations in sporadic MTC is around $8.8 \%$, and these authors confirmed that RET and RAS mutations were mutually exclusive. On the other hand, $40-60 \%$ of sporadic MTCs remained unassociated with any recognized genetic event.

The discovery of single strands of noncoding RNA in the human genome and their role in modulating gene expression at post-transcriptional level represent a great breakthrough in the postgenome sequencing era. MicroRNAs are small and bind to the 3 - -untranslated region of target genes, suppressing translation and/or causing mRNA degradation. That is why microRNA can play an important part in essential processes such as cell differentiation, growth, and cell death [9]. Investigating the role of microRNAs is an essential aspect of cancer research [10], and there has been a growing interest of late in how they may influence the pathogenesis and prognosis of MTC [11-15]. In particular, the role of miR-224 in cancer is under investigation and has yet to be clearly established. It seems to be a negative prognostic factor in lung adenocarcinoma, colorectal cancers, hepatocellular carcinoma, and cervical carcinoma [16-19]. On the other hand, several recent studies have identified its overexpression as a marker of a greater radio-sensitivity in glioblastoma and chemosensibility in prostatic carcinoma [20, 21]. These findings indicate that miR-224 has an indispensable role in cell proliferation, but also in the apoptosis of cancer cells, and the crucial balance between these two processes decides the miR-224 phenotype identifiable in tumor cells [22].

The aims of the present study on a large series of familial and sporadic cases of MTC were as follows: (a) to confirm our previous findings concerning miR-224 expression and its relationship with patient outcome; (b) to elucidate its relationship with the main molecular events responsible for MTC.

\section{Materials and Methods}

2.1. Patients. The cases considered were retrospectively selected from the electronic archives of the Surgical Pathology and Cytopathology Unit at the University of Padua, based on the elevated calcitonin levels and the diagnosis on MTC. All patients involved in this study gave their written informed consent, and the institute's ethical regulations on research on human tissues were followed.

The study concerned a consecutive series of 134 patients with MTC (107 sporadic and 27 familial; 54 males and 80 females; median age 59, range 5-87 years) collected from 2006 to 2015 with a median follow-up of 40 months (range
1-140 months). Serum Ct levels at diagnosis were available for most all the patients, who were considered biochemically cured if they had basal Ct levels below $10 \mathrm{pg} / \mathrm{ml}$ a year after primary surgery, and/or at the latest follow-up. Disease progression status was defined based on increasing disease burden, according to RECIST criteria, and/or on Ct/CEAdoubling times lower than 24 months. Patients were considered with stable disease if Ct/CEA-doubling times were higher than 24 months, without increasing disease burden [4].

At the end of the study period, $65 \%$ of patients $(86 / 133)$ were biochemically disease-free judging from their latest $\mathrm{Ct}$ test (which was unavailable for 1 patient). During the study period, $13 \%$ of the patients (17/134) had progressive disease, and 8 of them were treated with tyrosine kinase inhibitors (TKI). By the end of the study period, 7\% (9/132) had died of their disease.

2.2. RET and RAS Analysis. For each cancer sample, before DNA/RNA extraction, two experienced pathologists (F. G. and G. P.) analyzed a frozen section slightly colored by hematoxylin and eosin confirming MTC diagnosis and checking that at least $70 \%$ of cancer cells were present. DNA was extracted from tissue and from whole-blood samples using the DNeasy Blood and Tissue kit (Qiagen, Milano, Italy) according to the manufacturer's protocol in order to establish their mutational status and whether any mutations were germline or sporadic. Analyses were run for RET (NM_020975.4) (exons 5, 8, 10, 11, 13, 14, 15, and 16), N-RAS (NM_002524.3) (exons 2 and 3), K-RAS (NM_033360.2) (exons 2 and 3), and H-RAS (NM_005343.2) (exons 2 and 3 ) mutations by direct sequencing (bidirectionally, as standard practice in positive samples), as explained elsewhere [7,23]. When RET or RAS mutations were found, we confirmed the results by a new amplification PCR product and then bidirectionally sequencing.

\section{3. miR-224 Quantitative Real-Time Polymerase Chain} Reaction. Tissue cores were deparaffinized with xylene at $50^{\circ} \mathrm{C}$ for $3 \mathrm{~min}$. Total RNA extraction was done using the RecoverAll kit (Ambion, Austin, Texas, USA) according to the manufacturer's instructions. The NCode ${ }^{\mathrm{TM}}$ miRNA qRT-PCR method (Invitrogen, Carlsbad, California, USA) was used to detect and quantify mature hsa-miR-224 (primer: $5^{\prime}$-gca agt cac tag tgg ttc cgt $\mathrm{t}-3^{\prime}$ ) on a real-time LightCycler 480 instrument, according to the manufacturer's instructions (Roche, Milan, Italy). Normalization was performed with the small nuclear RNA U6B (RNU6B; primer: $5^{\prime}$-acg caaattcgtgaagcg $\left.\mathrm{tt}-3^{\prime}\right)$. Data were analyzed using the comparative cycle threshold (CT) method by LightCycler ${ }^{\circledR}$ Relative Quantification Software, 2001. All real-time reactions, including no template controls, were run in duplicate, as described elsewhere [24].

2.4. Statistical Analysis. All statistical analyses were performed using the MedCalc software (rel. 11.6.0). The Kolmogorov-Smirnov test was used to assess the normal distribution of each variable. After logarithmic transformation, the $t$-test and ANOVA were used to measure differences in miR-224 expression levels in different subgroups based on 
TABLE 1: Clinical, pathological, and molecular data on MTC cases.

\begin{tabular}{|c|c|}
\hline & MTC \\
\hline Total number & 134 \\
\hline \multicolumn{2}{|l|}{ Gender } \\
\hline Male & $54 / 134(37 \%)$ \\
\hline Female & $80 / 134(43 \%)$ \\
\hline Median age $(95 \% \mathrm{CI})$ & $58.5(55-61)$ \\
\hline Median follow-up in months (95\%CI) & $40(32-42)$ \\
\hline $\begin{array}{l}\text { Median calcitonin level in } \mathrm{pg} / \mathrm{ml} \text { at } \\
\text { diagnosis }(95 \% \mathrm{CI})\end{array}$ & $466(5.5-22931)$ \\
\hline \multicolumn{2}{|l|}{ Stage } \\
\hline I & $62 / 134(46 \%)$ \\
\hline II & $26 / 134(19 \%)$ \\
\hline III & $13 / 134(10 \%)$ \\
\hline IV & $33 / 134(25 \%)$ \\
\hline \multicolumn{2}{|l|}{ Lymph node involvement } \\
\hline Present & $46 / 134(34 \%)$ \\
\hline Absent & $88 / 134(66 \%)$ \\
\hline \multicolumn{2}{|l|}{ Distant metastases } \\
\hline Present & $12 / 134(9 \%)$ \\
\hline Absent & $122 / 134(91 \%)$ \\
\hline \multicolumn{2}{|l|}{ Germline RET mutation } \\
\hline Present & $27 / 134(20 \%)$ \\
\hline Absent & $107 / 134(80 \%)$ \\
\hline \multicolumn{2}{|l|}{ Sporadic RET mutation } \\
\hline Present & $39 / 107(36 \%)$ \\
\hline Absent & $68 / 107(64 \%)$ \\
\hline \multicolumn{2}{|l|}{ Sporadic RAS mutation } \\
\hline Present & $10 / 107(9 \%)$ \\
\hline Absent & $94 / 107(91 \%)$ \\
\hline \multicolumn{2}{|l|}{ Biochemical cure $^{1}$} \\
\hline Present & $86 / 133(65 \%)$ \\
\hline Absent & $35 / 133(35 \%)$ \\
\hline \multicolumn{2}{|l|}{ Disease progression } \\
\hline Present & $17 / 133(13 \%)$ \\
\hline Absent & $116 / 133(87 \%)$ \\
\hline Disease-related death $^{2}$ & 9/132 (7\%) \\
\hline
\end{tabular}

${ }^{1} \mathrm{Ct}$ level was unavailable for one patient. ${ }^{2}$ Two patients were lost to follow-up.

clinical/pathological variables (female versus male, different stages at diagnosis, lower versus higher Ct levels at diagnosis, biochemically cured status versus persistent disease, disease progression versus a stable disease, and death versus alive) and molecular features (RET/RAS status). The MannWhitney test and Kruskal-Wallis test for nonparametric data were used to correlate Ct levels with pathological and molecular data and patient outcome. Fisher's exact test was used to study the influence of somatic mutations on patient outcome in sporadic MTC cases. A rank correlation analysis was used to study the influence of miR-224 on CT levels at diagnosis. The Kaplan-Meier method was used to estimate the survival rates, and the log-rank test was used to assess the survival differences between the groups (the miR-224 levels were dichotomized as "high" or "low" based on the mean value of the variable). Multiple logistic regression was also used to ascertain the independent effect of miR-224 and the clinical and pathological features considered on the outcome of MTC patients. Differences were considered statistically significant when $p$ was less than 0.05 .

\section{Results}

Table 1 shows patients' clinical and pathological findings and their molecular RET/RAS status.

We initially examined the clinical/pathological differences in relation to serum Ct levels at diagnosis, then we considered the differences in miR-224 expression level in relation to the following: (a) clinical/pathological findings (CT level at diagnosis, nodal and distant involvement, and TNM stage); (b) patient's outcome; and (c) somatic RET and RAS mutations in sporadic MTCs.

As expected, higher serum Ct levels at diagnosis correlated positively with the presence of nodal and distant metastases, higher stage disease at diagnosis (for all variables, $p=0.0001$; $t$-test), persistent disease during the follow-up $(p=0.0001 ; t$-test $)$, and a higher risk of disease-related death $(p=0.0001 ; t$-test).

The expression levels of miR-224 were significantly associated with patients' main clinical and pathological findings and with their outcome: there was a statistically significant negative association between miR-224 expression level and serum Ct level at diagnosis: the higher the former, the lower the latter $(p=0.03, r=-0.3$; rank correlation). We found lower miR-224 values in patients with advanced stage disease at diagnosis $(p=0.001$; ANOVA): patients with nodal and distant metastases at diagnosis had significantly lower miR-224 levels ( $p=0.005$ and $p=0.0001$, resp.; $t$-test). We also found significantly lower miR-224 expression levels during the study period in patients with persistent disease, progressive disease, and fatal disease progression $(p=0.001$, $p=0.002$, and $p=0.0001$, resp.; $t$-test) (Figure 1). As expected from the Kaplan-Meier analysis and log-rank test, patients with a low miR-224 expression tended to have a shorter overall survival than those with a high miR-224 expression levels ( $p=0.005$; log-rank test) (Figure 2).

We found somatic RET mutations in $36 \%$ of cases of sporadic MTC (39/107), most of them located on exon 16 at codon 918 (M918T in 23/39; 59\%). In one patient $(2.6 \%$ ), RET mutation was on exon 8 (A513G), in 2 (5.1\%) on exon 10 (C609S, C618R), and in $8(20.5 \%)$ on exon 11 (2 with a C630R, 1 with a C630S, 2 with a C634Y, 1 with a C634W, 1 with a del613E, and 1 patient with a p.L629_L633del.). Five patients (12.8\%) had their RET mutation on exon 15 (3 with a A883F and 2 with a p.D898_E901del). Somatic RAS mutations were found in 10 sporadic MTC patients (10/107; 9\%): 3 had a mutation in K-RAS (two G12R and one G13R); one had a mutation in $N$-RAS (Q61L); and 6 had a mutation in H-RAS (two Q61R, one Q61K, one M72I, one G12R, and one was a novel G60D mutation, never previously reported in the literature). This last novel mutation warrants further study to clarify its role and importance, and the patient carrying this G60D mutation was consequently excluded from 


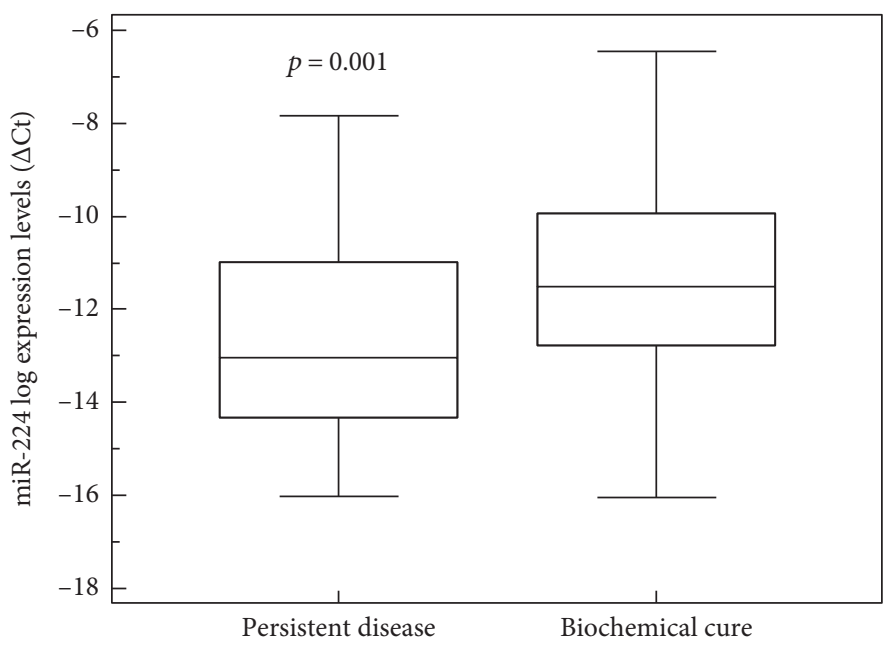

(a)

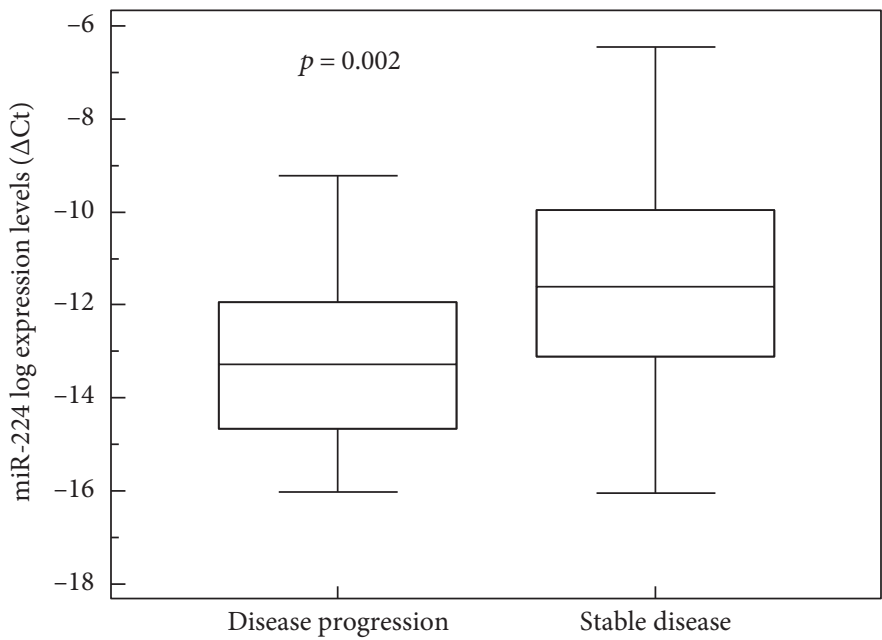

(b)

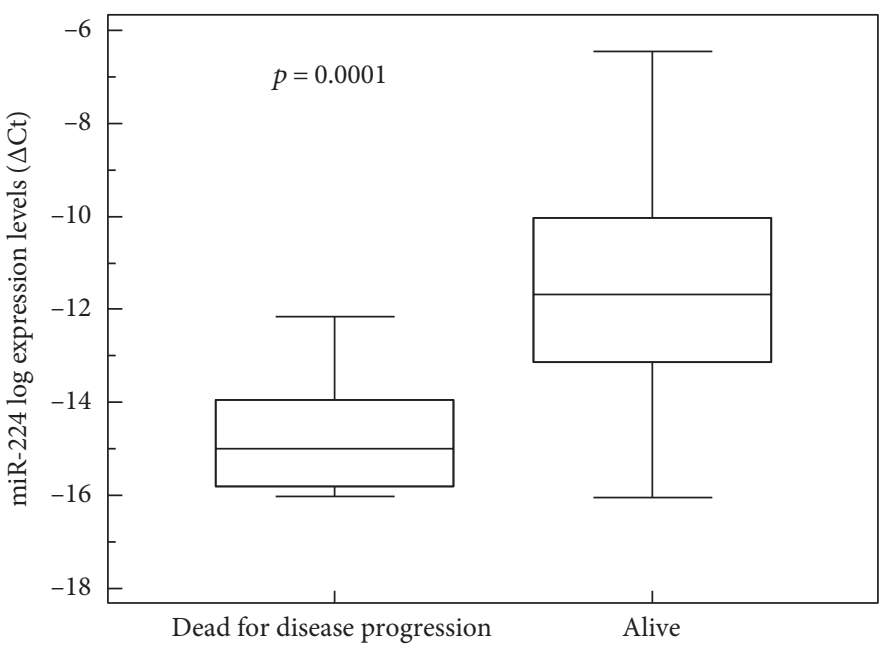

(c)

FIGURE 1: (a) Correlation between miR-224 expression level and persistent disease: lower levels of miR-224 were associated with higher risk of persistent (box-and-whisker plot, $p=0.001$ ). (b) Correlation between miR-224 expression level and disease progression: lower levels of miR-224 were associated with higher risk of disease progression (box-and-whisker plot, $p=0.002$ ). (c) miR-224 expression levels and death for disease progression. At the end of the follow-up, patients who died with progressive disease had lower miR-224 levels (box-and-whisker plot, $p=0.0001$ ). 


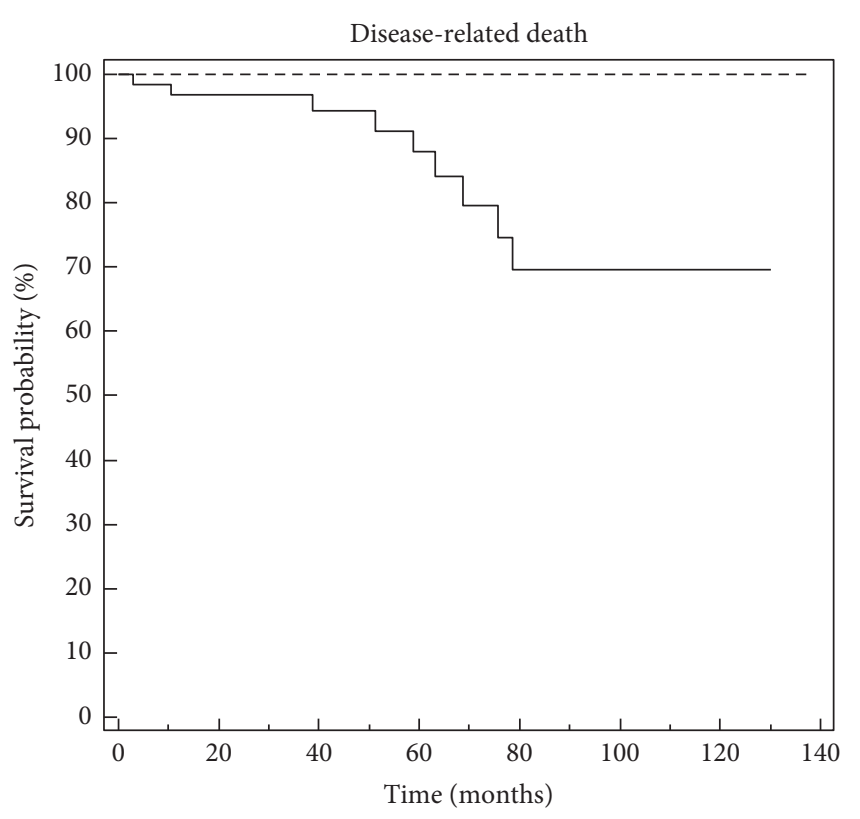

miR-224 levels

- Low

- - High

Figure 2: miR-224 and survival curve. On Kaplan-Meier analysis, patients with lower miR-224 expression levels tended to have a shorter survival than those with higher miR-224 levels. The miR-224 levels were dichotomized as "high" or "low" based on the mean value of the variable (log-rank test $p=0.005$ ).

the statistical analysis. Here again, we confirmed that $R A S$ and $R E T$ sporadic mutations were mutually exclusive.

In the sporadic cases, RET somatic mutations correlated with the following: the presence of nodal metastases $(p=0.02$, Fisher's exact test); distant metastases at diagnosis ( $p=0.01$, Fischer's exact test); and advanced stage at diagnosis ( $p=0.02$; Fisher's exact test). RET mutations also correlated with persistent disease at the end of the follow-up and an increased risk of disease-related death: the prevalence of RET mutations was significantly lower among patients who were cured than among those with persistent disease at the latest follow-up (28\% versus 50\%, $p=0.04$; Fisher's exact test), while it was much higher among patients who died of their disease than among those still alive $(78 \%$ versus $32 \%$, $p=0.02$; Fisher's exact test). We found no significant association between the presence of RET mutations and miR-224 expression levels. Furthermore, when we compared MTC outcome according to different RET mutations (M918T cases versus non-M918T ones), we found a significant association between the presence of the M918T mutation and persistent disease ( $p=0.02$, by chi-squared test). No other significant association was found.

When we considered the associations between the presence of RAS somatic mutations and the main clinical and pathological variables, we found no statistically significant correlations. However, all RAS-mutated patients were alive at the end of the study period: one had biochemical evidence of disease with stably low serum Ct levels (around
$45 \mathrm{ng} / \mathrm{L}$ ); the others were biochemically cured. We also found a significant positive association between RAS-mutated status and miR-224 expression levels: the presence of RAS mutations was associated with higher miR-224 levels $(p=0.007$; $t$-test), and the association was confirmed after the exclusion of RET-positive patients, too ( $p=0.03$; $t$-test), confirming that sporadic $R A S+/ R E T-M T C$ is a less aggressive phenotype (Figure 3). In addition, in Table 2, we reported clinical data based on $R E T+/ R A S$ - versus $R E T-/ R A S$ - versus $R E T-/ R A S+$ to clearly highlight the different outcomes due to the mutational profile.

Finally, our multivariate logistic regression analysis demonstrated that lower serum Ct levels at diagnosis and higher levels of miR-224 expression correlated independently with biochemical cure (OR 0.999, 95\%CI 0.9987-0.999 and OR $1.4,95 \% \mathrm{CI} 1.06-1.8$, resp.). Stage at diagnosis and miR-224 expression levels correlated independently with disease progression (OR 29.8, 95\%CI 3.7-243.3 and OR 0.7, 95\%CI $0.5-0.9$, resp.), but only miR-224 levels correlated independently with disease-related death (OR $0.3,95 \% \mathrm{CI} 0.1-0.6$ ).

\section{Discussion}

It has been estimated that more than 500 miRNAs are expressed in humans [25]. These miRNAs are believed to regulate the expression of nearly 5000 human genes or $30 \%$ of all human proteins. Recent evidence has shown that the interactions between miRNAs and their numerous mRNA targets may have important roles in gene control inside the cells and may be implicated in a variety of biological processes, such as cellular differentiation, proliferation, and apoptosis. By fine-adjusting gene expression, miRNAs may govern aspects that are crucial in determining cancer phenotypes (i.e., signaling, differentiation, invasion, and metastasis). They target both tumor suppressor and oncogenic pathways [26], and their altered expression carries great diagnostic and prognostic potential [27-29]. In addition, miRNAs are highly stable and can be detected reliably in archival clinical samples and cytology specimens and are therefore considered ideal candidate biomarkers [30].

miR-224 is a microRNA commonly dysregulated in most human cancers that affects crucial cellular processes and resides in chromosome Xq28 [22]. The intriguing role of miR-224 has yet to be fully elucidated. It may depend on the expression of miR-224 target genes in various cell types, since it can promote or inhibit cancer cell growth, depending on the histotype of the malignancy concerned. Some surveys recently suggested that miR-224 overexpression could play a significant part in promoting tumor cell proliferation and migration, with an oncogenic role. Yu et al. found miR-224 upregulation and AKT activation synergistically associated with tumor progression in hepatocellular carcinoma (HCC) [25]. Lan et al. found that HCV-induced low autophagy could lead to a high miR-224 expression with a tumorigenic effect [16]. Huang et al. suggested that miR-224 might have an important role in promoting the onset and progression of bone metastases from breast cancer [31]. miR-224 is an independent prognostic marker in cervical cancer and lung cancer too $[17,19,32]$. On the other hand, Mencia 


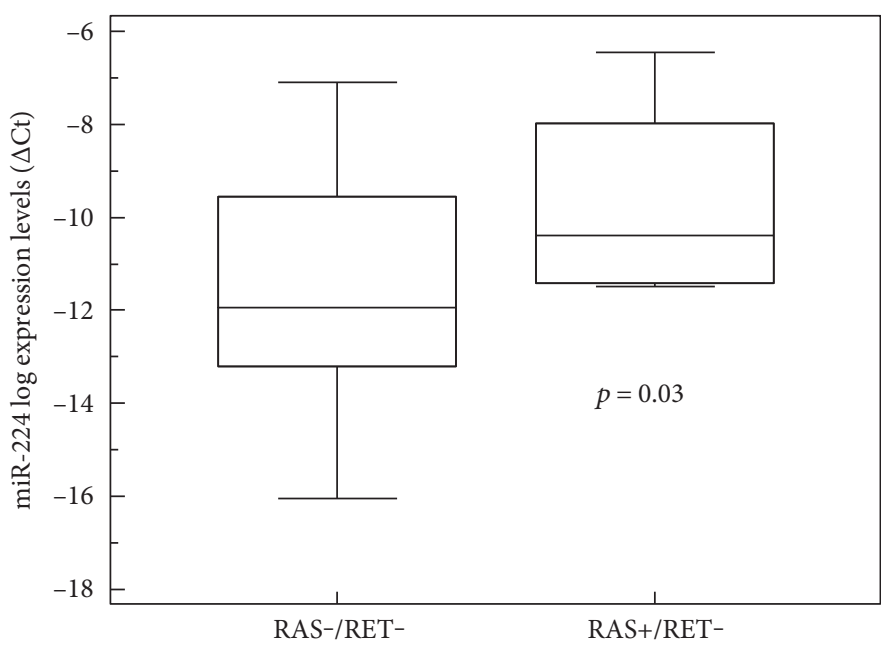

FIGURE 3: Correlation between miR-224 expression level and mutational status. The presence of a RAS mutation was associated with higher levels of miR-224 (box-and-whisker plot, $p=0.03$ ).

TABLe 2: Clinical data based on RET and RAS mutational status in sporadic MTCs.

\begin{tabular}{|c|c|c|c|c|}
\hline & $\begin{array}{c}\text { RET+/RAS- } \\
n=39 \\
(\%)\end{array}$ & $\begin{array}{c}\text { RET-/RAS- } \\
n=58 \\
(\%)\end{array}$ & $\begin{array}{c}\text { RAS +/RET- } \\
n=9^{1} \\
(\%)\end{array}$ & $p$ \\
\hline Median calcitonin level in $\mathrm{pg} / \mathrm{ml}$ at diagnosis $(n=106)$ & $700(83-22931)$ & $331(20-42300)$ & $402(61-747)$ & 0.01 \\
\hline \multicolumn{5}{|l|}{ Stage } \\
\hline $\mathrm{I}-\mathrm{II}(n=66)$ & $18(46 \%)$ & $41(71 \%)$ & $7(78 \%)$ & \multirow{2}{*}{0.03} \\
\hline III-IV $(n=40)$ & $21(54 \%)$ & $17(29 \%)$ & $2(22 \%)$ & \\
\hline Lymph node metastases $(n=40)$ & $21(54 \%)$ & $17(29 \%)$ & $2(22 \%)$ & 0.03 \\
\hline Biochemical cure $^{2}(n=64)$ & $18(46 \%)$ & $38(67 \%)$ & $8(89 \%)$ & 0.03 \\
\hline Disease progression $(n=15)$ & $9(24 \%)$ & $6(10 \%)$ & 0 & 0.08 \\
\hline Disease-related death ${ }^{3}(n=9)$ & $7(18 \%)$ & $2(4 \%)$ & 0 & 0.03 \\
\hline
\end{tabular}

${ }^{1}$ One patient with a novel RAS mutation was excluded from statistical analysis. ${ }^{2} \mathrm{Ct}$ level was unavailable for one patient. ${ }^{3}$ Two patients were lost to follow-up.

et al. demonstrated that miR-224 underexpression leads to insensitivity towards methotrexate, favoring a resistant phenotype [18]. In glioblastoma, miR-224 overexpression increases radiation sensitivity, thus improving outcomes, and patients with high miR-224 expression levels reportedly have a better overall survival [20]. Finally, miR22-4 downregulation was found to promote tumor progression in prostate cancer [33]. It is also worth mentioning the recent finding that, although miR-224 upregulation is a known negative prognostic factor in HCC, the high miR-224 phenotype in this cancer has been found associated with a better response to sorafenib, an inhibitor of several tyrosine kinase receptors, such as RET, RAF kinase, and vascular endothelial growth factor (VEGF) receptor, that is also used in MTC [34]. In the light of the above findings, and as demonstrated for other miRNAs, such as miR-221 and miR-222 [35-37], miR-224 seems to have a dual mode of action that depends on the type of cancer involved: it acts as an onco-miR in some cancers and as an oncosuppressor-miR in others, suggesting that different molecular targets and networks are regulated by miR224 in different neoplastic scenarios. As Croce put it, "before describing a miRNA as a tumor suppressor or an oncogene, it is necessary to specify in which cell or tissue, as cellular context is crucial for the function of miRNAs" [38].

To our knowledge, this is the first study to explore the expression profile of miR-224 in a large cohort of MTC patients, assessing its relationship with clinical, pathological, and molecular features. The literature currently provides little or no information on miR-224 expression levels in neuroendocrine tumors. A previous study of ours on a small series of 34 MTC patients found that miR-224 could represent a prognostic biomarker associated with a better outcome [11]. Our present findings confirmed as much, since miR224 expression levels were significantly lower in patients with distant metastases, persistent disease, and disease progression during their follow-up. More importantly, as already shown in glioblastoma tissues, we ascertained that miR-224 expression levels was significantly associated with patient survival. Our Kaplan-Meier analysis showed that patients with tumors showing a low miR-224 expression levels were likely to have a significantly shorter overall survival than those with a high miR-224 expression levels, strongly suggesting that low miR-224 expression levels is a marker of a poor prognosis in patients with MTC. In association with 
serum Ct levels at diagnosis and stage of disease, miR-224 emerged from our multivariate analysis as an independent prognostic marker.

Our findings also support the positive prognostic role of $R A S$ mutation found in a previous multicenter study [8]: all of our RAS-mutated patients were alive, and they had a less aggressive MTC phenotype and higher levels of miR-224 expression (although, based on our data, we cannot rule out the possibility of such associations being accidental). Finally, in line with other reports, we found that RET and RAS mutations were mutually exclusive [39-42].

RET activation stimulates multiple downstream pathways, promoting cell growth, proliferation, survival, and differentiation [43]. Two of the main pathways involved are the mitogen-activated protein kinase (MAPK) and the phosphoinositide 3-kinase (PI3K)/AKT pathways [43]. It remains to be seen whether RAS mutations can also lead to the activation of both of these signaling pathways in sporadic MTC, or whether there is a preferential activation pathway. Lyra et al. examined mTOR activation in a series of 87 MTCs (10 familial and 77 sporadic) and found RAS mutations significantly associated with a more intense expression of phospho-S6 ribosomal protein ( $\mathrm{p}-\mathrm{S} 6$, a downstream effector of mTOR) [44], pointing to an association between mTOR pathway activation and the presence of RAS mutations in MTC. We consequently conducted a recent study and demonstrated that MTC-harboring RAS mutations showed a preferential activation of the $\mathrm{PI} 3 \mathrm{~K} / \mathrm{Akt} / \mathrm{mTOR}$ pathway, revealed by an intense phospho-Akt reactivity pattern on Western blot analysis [24]. The different outcomes in RASversus RET-mutated patients could be due to these different activation pathways. The present study showed that, together with the presence of RAS mutations, higher miR-224 expression levels are also molecular markers of a favorable prognosis in sporadic MTC. Further studies are now needed to clarify whether these two factors are both implicated in the same signaling network.

In the clinical setting, Ct-doubling time and CEAdoubling time represent the most important markers for predicting the behavior of MTC [4], but these parameters change over time, being longer when the disease is in its early stages and shorter in later stages, when there is disease progression [8]. The molecular characterization of sporadic MTC at diagnosis, based on a search for somatic HRAS, KRAS, NRAS, or RET mutations, or miRNA expression profiling, will hopefully pave the way to a customized patient follow-up from the outset in the near future $[3,8]$. In addition, the discovery of miRNAs offers a novel mechanism for different treatment options and efforts to develop new modulators capable of inhibiting oncogenic miRNAs by using miRNA antagonists (anti-miRs), or by introducing a tumor suppressor miRNA mimetic to restore a loss of function [45-47]. Judging from our data, miR-224 acts as an oncosuppressor-miR in MTC and lower miR-224 expression levels serves as an independent prognostic molecular marker of a more aggressive disease that can identify patients at risk of progression and MTC-related death. Thanks to its stability and the reliability with which it can be assayed in different types of specimen (plasma, cytology, and frozen tissue),
miR-224 could represent an ideal marker in MTC, enabling an improvement in patients' risk stratification from the start.

\section{Conflicts of Interest}

The authors have no conflict of interest to disclose.

\section{Acknowledgments}

The authors would like to thank Frances Coburn for the text editing. This study was conducted using the resources of the Tissue Bank at the 1st Surgical Clinic, Padova University Hospital. The authors wish to thank Laura Zambonin for her excellent technical assistance.

\section{References}

[1] S. Leboulleux, E. Baudin, J. P. Travagli, and M. Schlumberger, "Medullary thyroid carcinoma," Clinical Endocrinology, vol. 61, no. 3, pp. 299-310, 2004.

[2] H. A. Sheikh, M. Tometsko, L. Niehouse et al., "Molecular genotyping of medullary thyroid carcinoma can predict tumor recurrence," The American Journal of Surgical Pathology, vol. 28, no. 1, pp. 101-106, 2004.

[3] R. T. Kloos, C. Eng, D. B. Evans et al., "Medullary thyroid cancer: management guidelines of the American Thyroid Association," Thyroid, vol. 19, no. 6, pp. 565-612, 2009.

[4] S. A. Wells Jr, S. L. Asa, H. Dralle et al., "Revised American Thyroid Association guidelines for the management of medullary thyroid carcinoma," Thyroid, vol. 25, no. 6, pp. 567-610, 2015.

[5] K. Frank-Raue, S. Rondot, and F. Raue, "Molecular genetics and phenomics of RET mutations: impact on prognosis of MTC," Molecular and Cellular Endocrinology, vol. 322, no. 1, pp. 2-7, 2010.

[6] R. Elisei, B. Cosci, C. Romei et al., "Prognostic significance of somatic RET oncogene mutations in sporadic medullary thyroid cancer. a 10-year follow-up study," The Journal of Clinical Endocrinology and Metabolism, vol. 93, no. 3, pp. 682-687, 2008.

[7] C. Mian, G. Pennelli, S. Barollo et al., "Combined RET and Ki-67 assessment in sporadic medullary thyroid carcinoma: a useful tool for patient risk stratification," European Journal of Endocrinology, vol. 164, no. 6, pp. 971-976, 2011.

[8] R. Ciampi, C. Mian, L. Fugazzola et al., "Evidence of a low prevalence of RAS mutations in a large medullary thyroid cancer series," Thyroid, vol. 23, no. 1, pp. 50-57, 2013.

[9] Z. Lichner, S. Mejia-Guerrero, M. Ignacak et al., "Pleiotropic action of renal cell carcinoma-dysregulated miRNAs on hypoxia-related signaling pathways," The American Journal of Pathology, vol. 180, no. 4, pp. 1675-1687, 2012.

[10] Z. Y. Lin, Y. Q. Huang, Y. Q. Zhang et al., "MicroRNA-224 inhibits progression of human prostate cancer by downregulating TRIB1," International Journal of Cancer, vol. 135, no. 3, pp. 541-550, 2014.

[11] C. Mian, G. Pennelli, M. Fassan et al., "MicroRNA profiles in familial and sporadic medullary thyroid carcinoma: preliminary relationships with RET status and outcome," Thyroid, vol. 22, no. 9, pp. 890-896, 2012.

[12] D. Abraham, N. Jackson, J. S. Gundara et al., "MicroRNA profiling of sporadic and hereditary medullary thyroid cancer 
identifies predictors of nodal metastasis, prognosis, and potential therapeutic targets," Clinical Cancer Research, vol. 17, no. 14, pp. 4772-4781, 2011.

[13] M. N. Nikiforova, G. C. Tseng, D. Steward, D. Diorio, and Y. E. Nikiforov, "MicroRNA expression profiling of thyroid tumors: biological significance and diagnostic utility," The Journal of Clinical Endocrinology and Metabolism, vol. 93, no. 5, pp. 1600-1608, 2008.

[14] J. Hudson, E. Duncavage, A. Tamburrino et al., "Overexpression of miR-10a and miR-375 and downregulation of YAP1 in medullary thyroid carcinoma," Experimental and Molecular Pathology, vol. 95, no. 1, pp. 62-67, 2013.

[15] L. Santarpia, G. A. Calin, L. Adam et al., "A miRNA signature associated with human metastatic medullary thyroid carcinoma," Endocrine-Related Cancer, vol. 20, no. 6, pp. 809-823, 2013.

[16] S. H. Lan, S. Y. Wu, R. Zuchini et al., "Autophagy-preferential degradation of MIR224 participates in hepatocellular carcinoma tumorigenesis," Autophagy, vol. 10, no. 9, pp. 16871689, 2014.

[17] S. N. Shen, L. F. Wang, Y. F. Jia, Y. Q. Hao, L. Zhang, and H. Wang, "Upregulation of microRNA-224 is associated with aggressive progression and poor prognosis in human cervical cancer," Diagnostic Pathology, vol. 8, no. 1, pp. 1-7, 2013.

[18] N. Mencia, E. Selga, V. Noé, and C. J. Ciudad, "Underexpression of miR-224 in methotrexate-resistant human colon cancer cells," Biochemical Pharmacology, vol. 82, no. 11, pp. 1572-1582, 2011.

[19] H. Wang, L. J. Zhu, Y. C. Yang, Z. X. Wang, and R. Wang, "MiR-224 promotes the chemoresistance of human lung adenocarcinoma cells to cisplatin via regulating G(1)/S transition and apoptosis by targeting p21(WAF1/CIP1)," British Journal of Cancer, vol. 111, no. 2, pp. 339-354, 2014.

[20] S. Upraity, S. Kazi, V. Padul, and N. V. Shirsat, "MiR-224 expression increases radiation sensitivity of glioblastoma cells," Biochemical and Biophysical Research Communications, vol. 448, no. 2, pp. 225-230, 2014.

[21] Y. Goto, R. Nishikawa, S. Kojima et al., "Tumour-suppressive microRNA-224 inhibits cancer cell migration and invasion via targeting oncogenic TPD52 in prostate cancer," FEBS Letters, vol. 588, no. 10, pp. 1973-1982, 2014.

[22] W. Chen, X. M. Fan, L. Mao et al., "MicroRNA-224: as a potential target for miR-based therapy of cancer," Tumour Biology, vol. 36, no. 9, pp. 6645-6652, 2015.

[23] G. Pennelli, F. Vianello, S. Barollo et al., "BRAF(K601E) mutation in a patient with a follicular thyroid carcinoma," Thyroid, vol. 21, no. 12, pp. 1393-1396, 2011.

[24] G. Pennelli, F. Galuppini, S. Barollo et al., "The PDCD4/ miR-21 pathway in medullary thyroid carcinoma," Human Pathology, vol. 46, no. 1, pp. 50-57, 2015.

[25] L. Yu, J. Zhang, X. Guo, Z. Li, and P. Zhang, "MicroRNA-224 upregulation and AKT activation synergistically predict poor prognosis in patients with hepatocellular carcinoma," Cancer Epidemiology, vol. 38, no. 4, pp. 408-413, 2014.

[26] A. Lujambio and S. W. Lowe, "The microcosmos of cancer," Nature, vol. 482, no. 7385, pp. 347-355, 2012.

[27] G. A. Calin, M. Ferracin, A. Cimmino, A. G. Di Leva, M. Shimizu, and S. E. Wojcik, "A microRNA signature associated with prognosis and progression in chronic lymphocytic leukemia," The New England Journal of Medicine, vol. 353, no. 17, pp. 1793-1801, 2005.
[28] J. Lu, G. Getz, E. A. Miska et al., "MicroRNA expression profiles classify human cancers," Nature, vol. 435, no. 7043, pp. 834-838, 2005.

[29] B. Minguez and A. Lachenmayer, "Diagnostic and prognostic molecular markers in hepatocellular carcinoma," Disease Markers, vol. 31, no. 3, pp. 181-190, 2011.

[30] A. Fassina, R. Cappellesso, F. Simonato, C. Lanza, A. Marzari, and M. Fassan, "Fine needle aspiration of non-small cell lung cancer: current state and future perspective," Cytopathology, vol. 23, no. 4, pp. 213-219, 2012.

[31] L. Huang, T. Dai, X. Lin et al., "MicroRNA-224 targets RKIP to control cell invasion and expression of metastasis genes in human breast cancer cells," Biochemical and Biophysical Research Communications, vol. 425, no. 2, pp. 127-133, 2012.

[32] R. Cui, W. Meng, H. L. Sun et al., "MicroRNA-224 promotes tumor progression in nonsmall cell lung cancer," Proceedings of the National Academy of Sciences of the United States of America, vol. 112, no. 31, pp. E4288-E4297, 2015.

[33] Y. Wan, Z. C. Zeng, M. Xi et al., "Dysregulated microRNA224/apelin axis associated with aggressive progression and poor prognosis in patients with prostate cancer," Human Pathology, vol. 46, no. 2, pp. 295-303, 2015.

[34] B. Gyongyosi, E. Vegh, B. Jaray et al., "Pretreatment microRNA level and outcome in sorafenib-treated hepatocellular carcinoma," The Journal of Histochemistry and Cytochemistry, vol. 62, no. 8, pp. 547-555, 2014.

[35] F. Fornari, L. Gramantieri, M. Ferracin et al., "MiR-221 controls CDKN1C/p57 and CDKN1B/p27 expression in human hepatocellular carcinoma," Oncogene, vol. 27, no. 43, pp. 5651-5661, 2008.

[36] C. le Sage, R. Nagel, D. A. Egan et al., "Regulation of the p27(Kip1) tumor suppressor by miR-221 and miR-222 promotes cancer cell proliferation," The EMBO Journal, vol. 26, no. 15, pp. 3699-3708, 2007.

[37] N. Felli, L. Fontana, E. Pelosi et al., "MicroRNAs 221 and 222 inhibit normal erythropoiesis and erythroleukemic cell growth via kit receptor down-modulation," Proceedings of the National Academy of Sciences of the United States of America, vol. 102, no. 50, pp. 18081-18086, 2005.

[38] C. M. Croce, "Causes and consequences of microRNA dysregulation in cancer," Nature Reviews. Genetics, vol. 10, no. 10, pp. 704-714, 2009.

[39] A. Boichard, L. Croux, A. Al Ghuzlan et al., "Somatic RAS mutations occur in a large proportion of sporadic RETnegative medullary thyroid carcinomas and extend to a previously unidentified exon," The Journal of Clinical Endocrinology and Metabolism, vol. 97, no. 10, pp. 2031-2035, 2012.

[40] M. J. Schlumberger, R. Elisei, L. Bastholt et al., "Phase II study of safety and efficacy of motesanib in patients with progressive or symptomatic, advanced or metastatic medullary thyroid cancer," Journal of Clinical Oncology, vol. 27, no. 23, pp. 3794-3801, 2009.

[41] M. M. Moura, B. M. Cavaco, A. E. Pinto, and V. Leite, "High prevalence of RAS mutations in RET-negative sporadic medullary thyroid carcinomas," The Journal of Clinical Endocrinology and Metabolism, vol. 96, no. 5, pp. 863-868, 2011.

[42] N. Agrawal, Y. Jiao, M. Sausen et al., "Exomic sequencing of medullary thyroid cancer reveals dominant and mutually exclusive oncogenic mutations in RET and RAS," The Journal of Clinical Endocrinology and Metabolism, vol. 98, no. 2, pp. 364-369, 2013. 
[43] C. Romei, R. Ciampi, and R. Elisei, "A comprehensive overview of the role of the RET proto-oncogene in thyroid carcinoma," Nature Reviews. Endocrinology, vol. 12, no. 4, pp. 192-202, 2016

[44] J. Lyra, J. Vinagre, R. Batista et al., "mTOR activation in medullary thyroid carcinoma with RAS mutation," European Journal of Endocrinology, vol. 171, no. 5, pp. 633-640, 2014.

[45] J. Krützfeldt, S. Kuwajima, R. Braich et al., "Specificity, duplex degradation and subcellular localization of antagomirs," Nucleic Acids Research, vol. 35, no. 9, pp. 2885-2892, 2007.

[46] A. G. Bader, D. Brown, and M. Winkler, "The promise of microRNA replacement therapy," Cancer Research, vol. 70, no. 18, pp. 7027-7030, 2010.

[47] G. Di Leva and C. M. Croce, "MiRNA profiling of cancer," Current Opinion in Genetics \& Development, vol. 23, no. 1, pp. 3-11, 2013. 


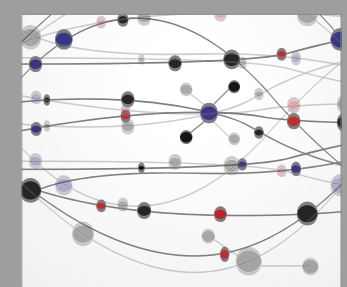

The Scientific World Journal
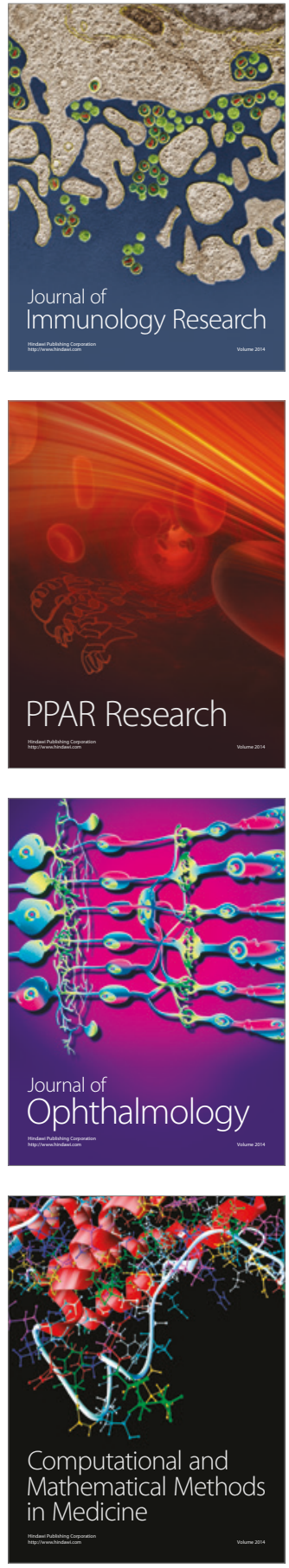

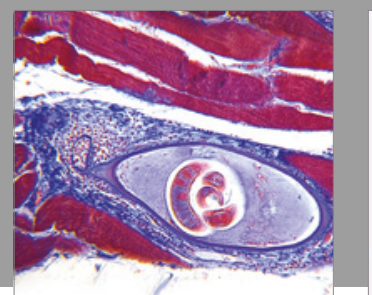

Gastroenterology Research and Practice
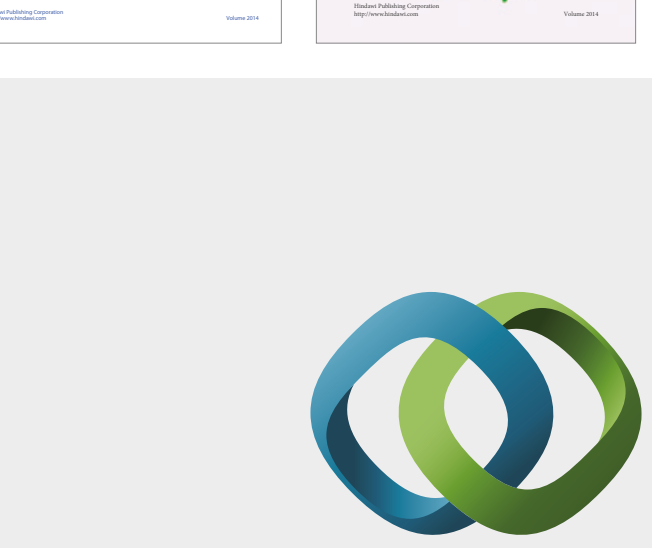

\section{Hindawi}

Submit your manuscripts at

https://www.hindawi.com
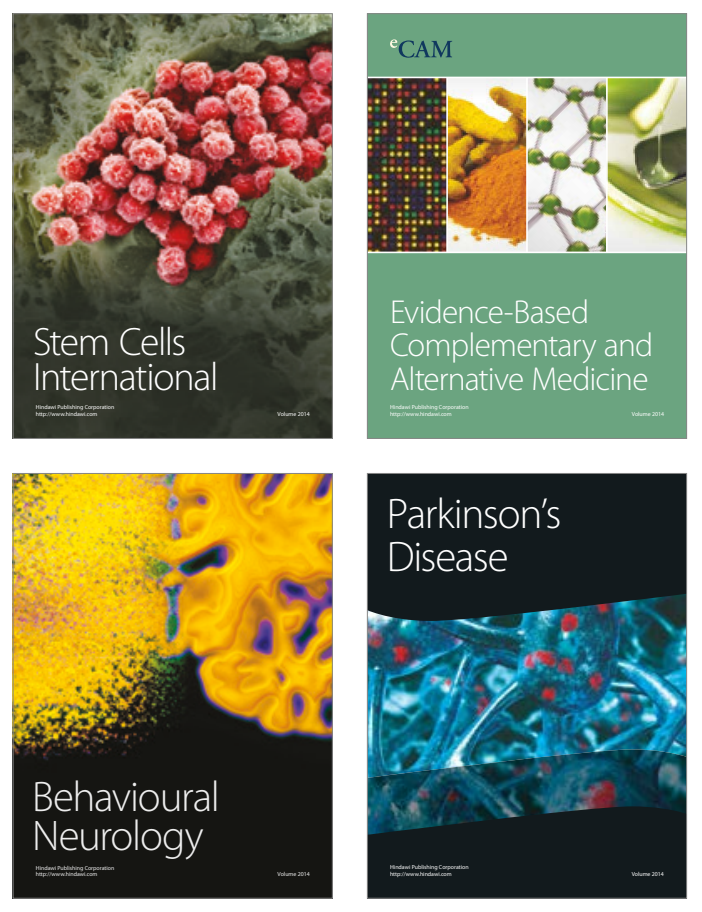
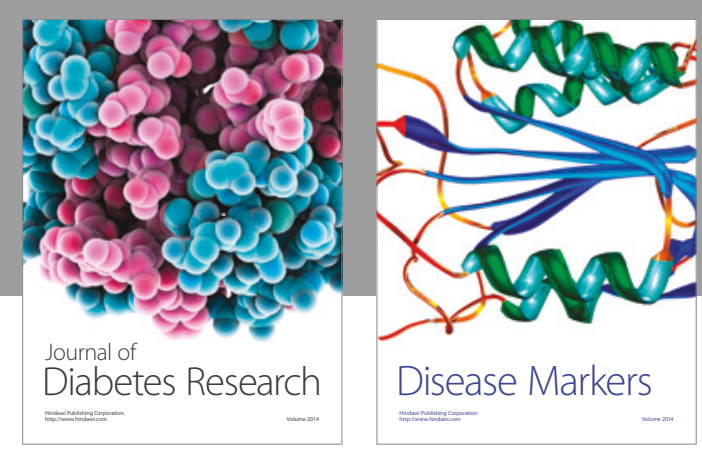

Disease Markers
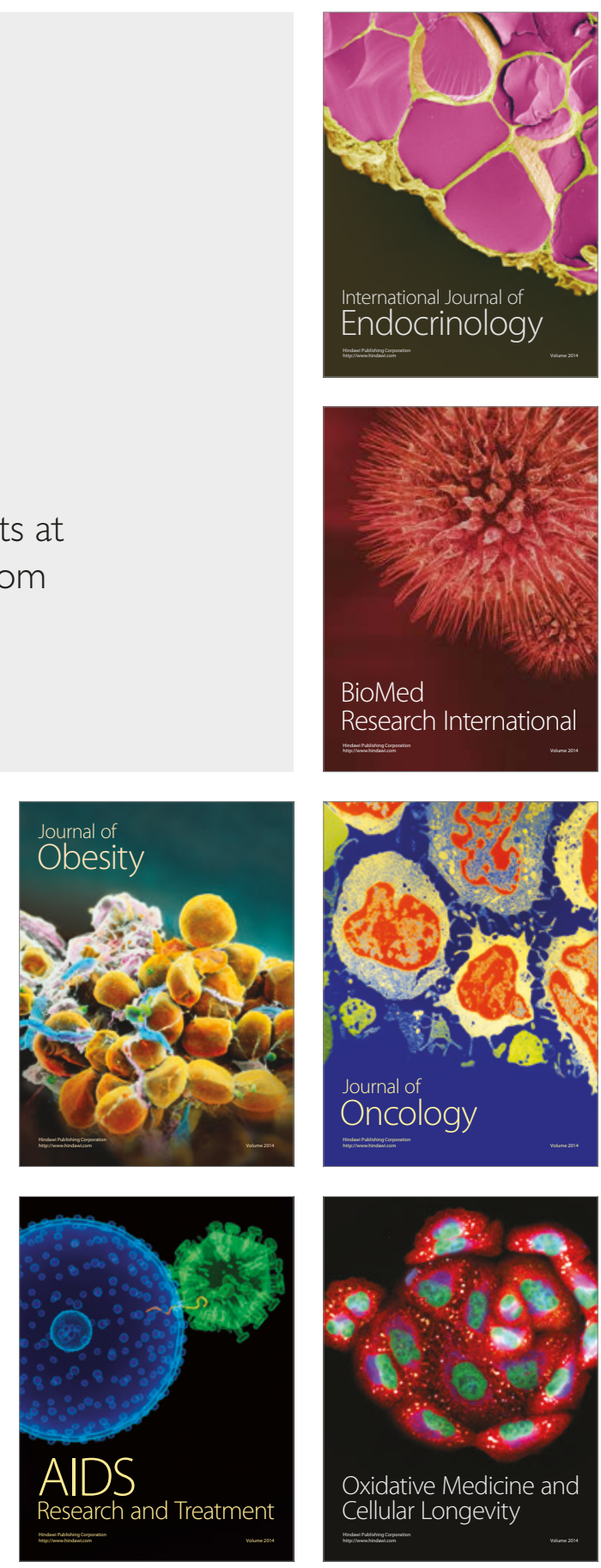